\section{CPC-086 MIGLUSTAT OFF-LABEL IN A PAEDIATRIC FORMULATION FOR A RARE METABOLIC DISEASE: EARLY INFANTILE GM1 GANGLIOSIDOSIS}

doi:10.1136/ejhpharm-2013-000276.543

'A Garzone, 'A Pompilio, 'F Ciuccarelli, 'E Andresciani, ${ }^{2} \mathrm{~A}$ Ficcadenti, ${ }^{3} \mathrm{M}$ De Meo, 'A Mannucci, 'P Marzioni, 'M Buccolini, 'L Carloni, ${ }^{3} \mathrm{~F}$ Pinto, 'V Moretti. 'AO Ospedali Riuniti - Presidio G. Salesi, Farmacia, Ancona, Italy; ${ }^{2}$ AO Ospedali Riuniti - Presidio G. Salesi, Centro Regionale Malattie Rare, Ancona, Italy; ${ }^{3}$ Università Degli Studi di Camerino, Scuola di Specializzazione in Farmacia Ospedaliera, Camerino, Italy

Background GM1 gangliosidosis, a rare metabolic disease, is an autosomal recessive lysosomal storage disorder caused by a deficiency of beta-galactosidase, and characterised by the generalised accumulation of GM1 ganglioside. The most severe form, called type I or early infantile, progressively destroys the neurons and the cells of the spinal cord.

No pharmacological treatment is available at present and children affected by GM1 type I usually do not survive past the age of three.

Miglustat (N-butyldeoxynojirimycin) is an iminosugar and functions as a competitive and reversible inhibitor of glucosylceramide synthase, the initial enzyme in reactions resulting in the synthesis of most glycosphingolipids. It is indicated in lysosomal storage diseases and it had positive results in GM2 gangliosidosis.

Purpose To describe our experience in the 12-month treatment with an individual formulation of miglustat, for a patient affected by GM1 gangliosidosis.

Materials and Methods In July 2011 Regione-Marche Centre for Rare Diseases involved our Hospital Pharmacy (authorization, galenic compounding) in supplying miglustat for an 18-month-old child with type I GM1 gangliosidosis, who was clearly showing severe signs of the pathology, lacking any other therapy.

Miglustat is off-label for this indication, so the approval of the Ethics Committee was necessary with the duty to report results every three months.

Personalized capsules were prepared for the hospital and home administration, with different doses (from $30 \mathrm{mg} / \mathrm{d}$ to $210 \mathrm{mg} / \mathrm{d}$ in 3 doses). The capsules were prepared from Zavesca, exclusively using mannitol as excipient: lactose is to be avoided in GM1 gangliosidosis

Results Miglustat provided surprising results, but only in the first four weeks of treatment. Frequent infections and seizures, typical of the disease, occurred later.

Conclusions The treatment is still being used, but after 12 months, we are considering stopping it. It would be important to compare other experiences in order to better evaluate the effectiveness of the treatment.

No conflict of interest.

\section{CPC-087 MONITORING ANTIEMETIC REGIMENS WITH APREPITANT IN CANCER PATIENTS}

doi:10.1136/ejhpharm-2013-000276.544

S Martinez, JJ Garcia, A Martiarena, M Nogales, V Goitia, MA Andres, C Martinez. Hospital Universitario de Alava, Pharmacy, Vitoria-Gasteiz, Spain

Background After including aprepitant in the hospital's pharmacotherapy guide 5 years ago, we decided to cheque if it is being used as defined by the Drug and Therapeutics Committee, which approved its use after the failure of other antiemetic treatments.

Purpose To evaluate the use of aprepitant in the Oncology Department of our hospital.

Materials and Methods We used the cytostatic prescription programme (Oncofarm Farmis-version 9.0.0.27) and dispensing programme (AS-400) to obtain the patients treated with aprepitant. The study period was from May 2010 to December 2011
Results A total of 52 patients (84\% women) were prescribed aprepitant during the study period. The average age was 49 years (age range: 19-69 years). The following data were collected: diagnosis and stage of disease, chemotherapy scheme, anti-emesis change cycle number, combination with radiotherapy and alcohol intake. $65 \%$ of patients had breast cancer followed by non-small lung cancer $(5 \%) .27 \%$ and $25 \%$ of cancers were in stages IA and IIA respectively. The most common chemotherapy scheme (55\%) for which the change of antiemetic therapy was seen, was FEC 500-100-500. $26 \%$ of patients started ondansetron 4 or $8 \mathrm{mg}$ before aprepitant was prescribed. The rest (74\%) received aprepitant directly after failing the first line antiemetic therapy. In $40 \%$ of patients the antiemetic regimen was changed to the study drug in cycle 2 and in $25 \%$ in cycle 3, demonstrating that aprepitant is not used as first-line antiemetic. Only 5 patients received radiotherapy combined with chemotherapy and only in 4 was alcohol intake recorded.

Conclusions In our hospital aprepitant is mainly used in chemotherapy regimens that include anthracyclines in combination with cyclophosphamide. It is prescribed after first line antiemetic regimen failure; meeting the indications established by Drug and Therapeutics Committee.

However, it would be advisable to cheque the antiemetic guidelines periodically for compliance with reference guides such as NCCN, ASCO, MASCC etc.

No conflict of interest.

\section{CPC-088 MONITORING OF THE CORRECT USE AND PRESCRIPTION METHODS OF PACLITAXEL ALBUMIN AND ERIBULIN AT THE EUROPEAN INSTITUTE OF ONCOLOGY}

doi:10.1136/ejhpharm-2013-000276.545

M Milani, P Paochi, E Omodeo Salè. European Institute of Oncology, Pharmacy, Milan, Italy

Background The use of innovative anticancer drugs in patients with various advanced tumours allows disease control with improved quality of life. This perspective must identify mechanisms to make additional costs arising from the most recent therapeutic advances affordable.

Purpose To evaluate the correct use and prescription methods of two new drugs in metastatic breast cancer in patients for whom the standard treatment for advanced disease has failed.

Materials and Methods Patients treated from April 2011 to May 2012 with Abraxane and from March 2012 to June 2012 with Halaven were extrapolated (start dates corresponded to the inclusion of drugs in the Hospital Therapeutic Handbook). For each patient individual doses were extrapolated and integrated with clinical data reported in the medical records.

Results 25 patients were treated with Abraxane, 10 with Halaven, while 3 received both treatments. Of the 25 patients treated with Abraxane, $9(36 \%)$ received the drug after at least 10 lines of treatment, 11 (44\%) after 6-9 lines, $5(20 \%)$ after 1-5 lines. 16 patients were treated according to SPC: $260 \mathrm{mg} / \mathrm{m}^{2} /$ day $1 \mathrm{q} 21$ (64\%), 9 with an off-label schedule: $100 \mathrm{mg} / \mathrm{m}^{2} /$ day $1.8 \mathrm{q} 21$ (36\%). Furthermore it was found that a dose reduction not corresponding to what was reported in the SmPC was often used. Among patients treated with Halaven, $8(80 \%)$ had received $8-15$ prior lines of treatment, only 2 $(20 \%)$ began treatment in $3 \mathrm{rd}-4$ th line. Among patients who went into progression (17 patients, $68 \%$ ), only $18 \%$ reached 10 doses, $6 \%$ achieved 7-9 doses, 23\% 4-6 doses and the majority (53\%) did not exceed 1-3 doses of the medicine.

Conclusions The evaluation of these data suggests the need for a more accurate selection of patients based on predictive baseline characteristics or early indicators of activities and the implementation of post-registration studies to confirm the efficacy and safety of these drugs.

No conflict of interest. 